\title{
Annexes au dossier thématique
}

\section{(2) OpenEdition}

1 Journals

Édition électronique

URL : http://journals.openedition.org/plc/228

DOI : $10.4000 /$ plc. 228

ISSN : 2117-5209

Éditeur

L'Harmattan

\section{Édition imprimée}

Date de publication : 15 décembre 2007

Pagination : 179-195

ISBN : 2-296-01986-2

ISSN : $1279-8657$

\section{Référence électronique}

"Annexes au dossier thématique », Pouvoirs dans la Caraïbe [En ligne], 15 | 2007, mis en ligne le 06 mai 2011, consulté le 10 décembre 2020. URL : http://journals.openedition.org/plc/228 ; DOI : https:// doi.org/10.4000/plc.228 


\section{ANNEXES}

ANNEXE I - Résultats des élections régionales de mars 2004 (Guadeloupe, Martinique)

ANNEXE II - Résultats de la consultation du 7 décembre (Guadeloupe, Martinique, Saint-Barthélemy, Saint-Martin)

ANNEXE III - La Constitution révisée (révision du 28 mars 2003)

ANNEXE IV - La « Déclaration de Basse-Terre » 


\section{ANNEXE I}

\section{RESULTATS DES ELECTIONS REGIONALES DE MARS 2004 (GUADELOUPE, MARTINIQUE)}

a) Guadeloupe

$1^{\text {er }}$ Tour (21 mars 2004)

\begin{tabular}{|l|c|c|c|}
\hline & Nombre & $\%$ Inscrits & \\
Inscrits & 289664 & $100,00 \%$ & \\
Abstention & 131693 & $45,46 \%$ & \\
& 157971 & $54,54 \%$ & \\
& & & \\
Votants & Nombre & $\%$ Votants & \\
Exprimés & 9031 & $5,72 \%$ & \\
& 123406 & $94,51 \%$ & \\
Liste conduite par & & & \\
Mme Octavie LOSIO & Nuance & Voix & $\%$ Exprimés \\
M. Jean-Marie NOMERTIN & LDG & 3085 & $2.07 \%$ \\
M. Ary BROUSSILLON & LDG & 1759 & $1.18 \%$ \\
M. Victorin LUREL & LRG & 5874 & $3.94 \%$ \\
Mme Juliette NUBRET NÉE ADONICAM & LGA & 65962 & $44.29 \%$ \\
M. Harry DURIMEL & LDD & 2302 & $1.55 \%$ \\
M. Daniel MARSIN & LVE & 4306 & $2.89 \%$ \\
Mme Lucette MICHAUX-CHEVRY & LGA & 8158 & $5.48 \%$ \\
M. Gérard LAURIETTE & LDR & 56024 & $37.62 \%$ \\
\hline
\end{tabular}

Source : Ministère de l'intérieur 
$2^{\text {ème }}$ Tour (28 mars 2004)

\begin{tabular}{|l|c|c|c|c|}
\hline Inscrits & Nombre & \% Inscrits & & \\
Abstention & 286899 & & & \\
Votants & 107781 & $37.57 \%$ & & \\
& 179178 & $62.43 \%$ & & \\
& Nombre & $\%$ Votants & & \\
Blancs Nuls & 7711 & $4.30 \%$ & & \\
Exprimés & 171407 & 95.70 & & \\
& & & & \\
Liste conduite par & Nuance & Voix & $\%$ Exprimés & Sièges \\
M. Victorin LUREL & LRA & 99805 & $58.23 \%$ & 29 \\
Mme Lucette MICHAUX-CHEVRY & LDR & 71602 & $41.77 \%$ & 12 \\
\hline
\end{tabular}

Source : Ministère de l'intérieur

Grille des nuances :

LDD : Liste Divers Droite

LDG : Liste Divers Gauche

LDR : Liste de droite

LGA : Liste d'Union de la Gauche

LRG : Liste régionaliste

LVE : Liste des Verts 
b) Martinique

$1^{\text {er }}$ Tour (21 mars 2004)

\begin{tabular}{|l|c|c|c|}
\hline & Nombre & $\%$ Inscrits & \\
Inscrits & 271922 & & \\
Votentionts & 141343 & $51,98 \%$ & \\
& 130579 & $48,02 \%$ & \\
& Nombre & $\%$ Votants & \\
Blancs \& Nuls & 7173 & $5,49 \%$ & \\
Exprimés & 123406 & $94,51 \%$ & \\
& & & \\
Liste conduite par & Nuance & Voix & $\%$ Exprimés \\
M. Alfred MARIE-JEANNE & LRG & 46006 & $37,28 \%$ \\
M. Miguel LAVENTURE & LDR & 17177 & $13,92 \%$ \\
M. Pierre PETIT & LDR & 8367 & $6,78 \%$ \\
Mme Madeleine DE GRANDMAISON & LDG & 21232 & $17,20 \%$ \\
M. Jean CRUSOL & LDG & 5864 & $4,75 \%$ \\
M. Pierre Jean SAMOT & LDG & 19994 & $16,20 \%$ \\
Mme Ghislaine JOACHIM-ARNAUD & LXG & 3866 & $3,13 \%$ \\
M. Max Auguste DUFRENOT & LRG & 900 & $0,73 \%$ \\
\hline
\end{tabular}

Source : Ministère de l'intérieur 
$2^{\text {ème }}$ Tour (28 mars 2004)

\begin{tabular}{|l|r|r|r|c|}
\hline Inscrits & Nombre & $\%$ Inscrits & & \\
Abstention & 271920 & & & \\
Votants & 127878 & $47,03 \%$ & & \\
& 144042 & $52,97 \%$ & & \\
& Nombre & \% Votants & & \\
& & & & \\
Blancs Nuls & 139257 & $3,32 \%$ & & \\
Exprimés & $96,68 \%$ & & \\
& Nuance & Voix & \% Exprimés & Sièges \\
Liste conduite par & & & & \\
& LRG & 74860 & $53,76 \%$ & 28 \\
M. Alfred MARIE-JEANNE & LDR & 21227 & $15,24 \%$ & 4 \\
M. Miguel LAVENTURE & LDG & 43170 & $31,00 \%$ & 9 \\
Mme Madeleine DE GRANDMAISON & & & \\
\hline
\end{tabular}

Source : Ministère de l'intérieur

Grille des nuances :

LDD : Liste Divers Droite

LDG : Liste Divers Gauche

LDR : Liste de droite

LGA : Liste d'Union de la Gauche

LRG : Liste régionaliste

LRG : Liste d'extrême gauche 


\section{ANNEXE II \\ RESULTATS DE LA CONSULTATION DU 7 DECEMBRE 2003 \\ (Guadeloupe, Martinique, Saint-Barthélemy, Saint-Martin).}

Consultation locale - Antilles

\section{QUESTION :}

"Approuvez-vous le projet de création d'une collectivité territoriale demeurant régie par l'article 73 de la Constitution, et donc par le principe de l'identité législative avec possibilité d'adaptations, et se substituant au département et à la région dans les conditions prévues par cet article?»

\section{RESULTATS :}

\begin{tabular}{|c|c|}
\hline $\begin{array}{l}\text { Guadeloupe } \\
\text { Nombre d'électeurs : 284.807 } \\
\text { Votants : } 142630(50,33 \%) \\
\text { BN : } 7444 \\
\text { Exprimés : } 135186 \\
\text { OUI : } 36526 \\
\text { NON : } 98660(\mathbf{7 2 . 9 8 \% )}\end{array}$ & $\begin{array}{l}\text { Martinique } \\
\text { Nombre d'électeurs : } 265056 \\
\text { Votants : } 116904(44,11 \%) \\
\text { BN : } 8565 \\
\text { Exprimés : } 108368 \\
\text { OUI : } 53667 \\
\text { NON : } \mathbf{5 4 7 0 1}(\mathbf{5 0 . 4 8 \% )}\end{array}$ \\
\hline $\begin{array}{l}\text { Saint-Martin } \\
\text { Nombre d'électeurs : } 13.413 \\
\text { Votants : } 5926(44,18 \%) \\
\text { BN : } 281 \\
\text { Exprimés : } 5645 \\
\text { OUI : 4300 } \\
\text { NON : } 1345\end{array}$ & $\begin{array}{l}\text { Saint-Barthélemy } \\
\text { Nombre d'électeurs : } 3.697 \\
\text { Votants : } 2910 \\
\text { BN : } 58 \\
\text { Exprimés : } 2852 \\
\text { OUI : 2724 } \\
\text { NON : } 128\end{array}$ \\
\hline
\end{tabular}




\section{ANNEXE III}

LA CONSTITUTION REVISEE (REVISION DU 28 MARS 2003)

a) Tableau récapitulatif des principales modifications

ARTICLE 72 DE LA CONSTITUTION

\begin{tabular}{|c|c|c|}
\hline CONCERNANT & ANCIEN & NOUVEAU \\
\hline $\begin{array}{l}\text { Enumération des } \\
\text { collectivités } \\
\text { territoriales }\end{array}$ & $\begin{array}{l}\text { Communes } \\
\text { Départements } \\
\text { Territoires d'outre-mer } \\
\text { Collectivités nouvelles } \\
\text { créées par la loi }\end{array}$ & $\begin{array}{l}\text { Communes } \\
\text { Départements } \\
\text { Régions } \\
\text { Collectivités à statut } \\
\text { particulier } \\
\text { Collectivités d'outre-mer } \\
\text { Collectivités nouvelles } \\
\text { créées par la loi } \\
\text { Nouvelles collectivités } \\
\text { remplaçant des } \\
\text { collectivités existantes }\end{array}$ \\
\hline $\begin{array}{l}\text { Pouvoirs des } \\
\text { collectivités } \\
\text { territoriales }\end{array}$ & $\begin{array}{l}\text { S'administrer librement } \\
\text { par des Conseils élus }\end{array}$ & $\begin{array}{l}\text { S'administrer librement } \\
\text { par des Conseils élus } \\
\text { Exercer un pouvoir } \\
\text { réglementaire }\end{array}$ \\
\hline $\begin{array}{l}\text { Domaines dans } \\
\text { lesquels les } \\
\text { collectivités } \\
\text { territoriales peuvent } \\
\text { exercer leurs } \\
\text { compétences }\end{array}$ & $\begin{array}{l}\text { Les domaines d'action de } \\
\text { chaque collectivité } \\
\text { territoriale sont précisés } \\
\text { par la loi }\end{array}$ & $\begin{array}{l}\text { Les domaines sont } \\
\text { précisés par la loi } \\
\text { Des expériences peuvent } \\
\text { être faites avant de } \\
\text { confier de nouveaux } \\
\text { domaines de compétence } \\
\text { à certaines collectivités } \\
\text { La répartition des } \\
\text { domaines de compétence } \\
\text { doit rechercher } \\
\text { l'efficacité et la } \\
\text { proximité par rapport aux } \\
\text { citoyens }\end{array}$ \\
\hline $\begin{array}{l}\text { Rapports entre les } \\
\text { collectivités } \\
\text { territoriales }\end{array}$ & & $\begin{array}{l}\text { Pas de supériorité de } \\
\text { certaines collectivités sur } \\
\text { d'autres } \\
\text { Pour faciliter les actions } \\
\text { communes, la loi désigne } \\
\text { un "chef de file" }\end{array}$ \\
\hline
\end{tabular}




\begin{tabular}{|l|l|l|}
\hline $\begin{array}{l}\text { Rôle du } \\
\text { représentant de } \\
\text { l'Etat dans les } \\
\text { collectivités }\end{array}$ & $\begin{array}{l}\text { Défendre les intérêts } \\
\text { nationaux }\end{array}$ & $\begin{array}{l}\text { Défendre les intérêts } \\
\text { nationaux }\end{array}$ \\
& Exercer un contrôle & Exercer un contrôle \\
administratif sur les & administratif sur les \\
& décisions des & décisions des collectivités \\
& collectivités & territoriales \\
& Assurer le respect des & Assurer le respect des \\
& lois & lois \\
& Coordonner les services & Réaffirmation du rôle de \\
& déconcentrés & $\begin{array}{l}\text { coordination des services } \\
\text { déconcentrés }\end{array}$ \\
\hline
\end{tabular}


ARTICLE 73 DE LA CONSTITUTION

\begin{tabular}{|c|c|c|}
\hline CONCERNANT & ANCIEN & NOUVEAU \\
\hline Collectivités concernées & $\begin{array}{l}\text { Départements situés outre- } \\
\text { mer }\end{array}$ & $\begin{array}{l}\text { Départements situés outre- } \\
\text { mer } \\
\text { Régions situées outre-mer } \\
\text { Collectivité territoriale } \\
\text { nouvelle située outre-mer } \\
\text { se substituant à la région et } \\
\text { au département }\end{array}$ \\
\hline $\begin{array}{l}\text { Modalités d'application } \\
\text { des textes adoptés par les } \\
\text { autorités étatiques (lois, } \\
\text { décrets, arrêtés) }\end{array}$ & $\begin{array}{l}\text { Même régime que pour les } \\
\text { départements } \\
\text { métropolitains (sous- } \\
\text { entendu) } \\
\text { Adaptations possibles par } \\
\text { l'autorité qui adopte le } \\
\text { texte, nécessitées par leur } \\
\text { situation particulière }\end{array}$ & $\begin{array}{l}\text { De plein droit } \\
\text { (= automatique) } \\
\text { Adaptations possibles par } \\
\text { l'autorité qui adopte le } \\
\text { texte, tenant à leurs } \\
\text { caractéristiques et } \\
\text { contraintes particulières } \\
\text { Adaptations décidées } \\
\text { localement par les } \\
\text { collectivités territoriales } \\
\text { dans les domaines où elles } \\
\text { sont compétentes après } \\
\text { autorisation donnée par le } \\
\text { Parlement }\end{array}$ \\
\hline $\begin{array}{l}\text { Pouvoir local d'adopter } \\
\text { des règles }\end{array}$ & - & $\begin{array}{l}\text { Possibilité d'adopter des } \\
\text { règles dans les domaines } \\
\text { de compétence réservés au } \\
\text { Parlement, mais de façon } \\
\text { très limitée et après } \\
\text { autorisation du Parlement }\end{array}$ \\
\hline $\begin{array}{l}\text { Possibilité de remplacer } \\
\text { une ou plusieurs } \\
\text { collectivités par une } \\
\text { collectivité nouvelle ou } \\
\text { d'avoir un seul groupe } \\
\text { d'élus pour s'occuper de } \\
\text { deux collectivités }\end{array}$ & $\begin{array}{l}\text { Il fallait maintenir le } \\
\text { département } \\
\text { Les élus d'une Assemblée } \\
\text { unique s'occupant du } \\
\text { département et de la région } \\
\text { devaient être élus par } \\
\text { canton } \\
\text { Les compétences du } \\
\text { département (sauf celles } \\
\text { spécifiques à l'outre-mer) } \\
\text { ne pouvaient pas être } \\
\text { transférées à la région. }\end{array}$ & $\begin{array}{l}\text { Avec le consentement des } \\
\text { électeurs, il est possible : } \\
\text { - Soit de remplacer le } \\
\text { département et la région } \\
\text { par une collectivité unique } \\
\text { nouvelle } \\
\text { - Soit de confier au même } \\
\text { groupe d'élus le soin d'agir } \\
\text { tantôt au nom de la région, } \\
\text { tantôt au nom du } \\
\text { département. (Assemblée } \\
\text { unique) } \\
\text { Le Parlement est libre de } \\
\text { choisir le mode d'élection } \\
\text { des membres de } \\
\text { l'Assemblée unique ou des } \\
\text { élus de la collectivité } \\
\text { unique. }\end{array}$ \\
\hline
\end{tabular}


ARTICLE 74 DE LA CONSTITUTION

\begin{tabular}{|c|c|c|}
\hline CONCERNANT & ANCIEN & NOUVEAU \\
\hline $\begin{array}{l}\text { Modalités d'application } \\
\text { des textes nationaux }\end{array}$ & $\begin{array}{l}\text { Sur mention expresse } \\
\text { (sous-entendu) }\end{array}$ & $\begin{array}{l}\text { Sur mention expresse } \\
\text { (sous-entendu) }\end{array}$ \\
\hline $\begin{array}{l}\text { Texte qui définit leur } \\
\text { statut }\end{array}$ & $\begin{array}{l}\text { Une loi organique, après } \\
\text { consultation de leur } \\
\text { Assemblée, complétée par } \\
\text { des lois ordinaires }\end{array}$ & $\begin{array}{l}\text { Une loi organique, après } \\
\text { consultation de leur } \\
\text { Assemblée, complétée par } \\
\text { des lois ordinaires }\end{array}$ \\
\hline $\begin{array}{l}\text { Contenu des lois } \\
\text { organiques propres à } \\
\text { chaque collectivité d'outre- } \\
\text { mer }\end{array}$ & $\begin{array}{l}\text { Compétences de leurs } \\
\text { collectivités }\end{array}$ & $\begin{array}{l}\text { Pour toutes : } \\
\text { Les conditions } \\
\text { d'application des textes } \\
\text { nationaux } \\
\text { Les compétences de la } \\
\text { collectivité, mises à part } \\
\text { celles qui sont réservées à } \\
\text { l'Etat } \\
\text { L'organisation et le } \\
\text { fonctionnement de la } \\
\text { collectivité } \\
\text { Les conditions dans } \\
\text { lesquelles elle est consultée } \\
\text { sur différentes questions } \\
\text { Pour celles qui sont } \\
\text { dotées de l'autonomie } \\
\text { (Polynésie) : } \\
\text { Le contrôle du Conseil } \\
\text { d'Etat sur les actes de la } \\
\text { collectivité } \\
\text { Les possibilités de prendre } \\
\text { des mesures en faveur de la } \\
\text { population de la } \\
\text { collectivité en matière } \\
\text { d'emploi, de droit } \\
\text { d'établissement, d'exercice } \\
\text { d'une activité } \\
\text { professionnelle ou de } \\
\text { protection du patrimoine } \\
\text { foncier } \\
\text { Les possibilités } \\
\text { d'association à l'exercice } \\
\text { des compétences de l'Etat } \\
\end{array}$ \\
\hline
\end{tabular}




\section{b) Articles révisés de la Constitution}

Article 1. La France est une République indivisible, démocratique et sociale. Elle assure l'égalité devant la loi de tous les citoyens sans distinction d'origine, de race ou de religion. Elle respecte toutes les croyances. Son organisation est décentralisée.

Article 37. Les matières autres que celles qui sont du domaine de la loi ont un caractère réglementaire.

Les textes de forme législative intervenus en ces matières peuvent être modifiés par décrets pris après avis du Conseil d'Etat. Ceux de ces textes qui interviendraient après l'entrée en vigueur de la présente Constitution ne pourront être modifiés par décret que si le Conseil constitutionnel a déclaré qu'ils ont un caractère réglementaire en vertu de l'alinéa précédent.

Article 37-1. La loi et le règlement peuvent comporter, pour un objet et une durée limités, des dispositions à caractère expérimental.

Article 38. Le Gouvernement peut, pour l'exécution de son programme, demander au Parlement l'autorisation de prendre par ordonnances, pendant un délai limité, des mesures qui sont normalement du domaine de la loi.

Les ordonnances sont prises en Conseil des ministres après avis du Conseil d'Etat. Elles entrent en vigueur dès leur publication mais deviennent caduques si le projet de loi de ratification n'est pas déposé devant le Parlement avant la date fixée par la loi d'habilitation.

A l'expiration du délai mentionné au premier alinéa du présent article, les ordonnances ne peuvent plus être modifiées que par la loi dans les matières qui sont du domaine législatif.

Article 72. Les collectivités territoriales de la République sont les communes, les départements, les régions, les collectivités à statut particulier et les collectivités d'outre-mer régies par l'article 74. Toute autre collectivité territoriale est créée par la loi, le cas échéant en lieu et place d'une ou de plusieurs collectivités mentionnées au présent alinéa.

Les collectivités territoriales ont vocation à prendre les décisions pour l'ensemble des compétences qui peuvent le mieux être mises en œuvre à leur échelon.

Dans les conditions prévues par la loi organique, et sauf lorsque sont en cause les conditions essentielles d'exercice d'une liberté publique ou d'un droit constitutionnellement garanti, les collectivités territoriales ou leurs groupements peuvent, lorsque, selon le cas, la loi ou le règlement l'a prévu, déroger, à titre expérimental et pour un objet et une durée limités, aux dispositions législatives ou réglementaires qui régissent l'exercice de leurs compétences. 
Aucune collectivité territoriale ne peut exercer une tutelle sur une autre. Cependant, lorsque l'exercice d'une compétence nécessite le concours de plusieurs collectivités territoriales, la loi peut autoriser l'une d'elles ou un de leurs groupements à organiser les modalités de leur action commune.

Dans les collectivités territoriales de la République, le représentant de l'Etat, représentant de chacun des membres du Gouvernement, a la charge des intérêts nationaux, du contrôle administratif et du respect des lois.

Article 72-1. La loi fixe les conditions dans lesquelles les électeurs de chaque collectivité territoriale peuvent, par l'exercice du droit de pétition, demander l'inscription à l'ordre du jour de l'assemblée délibérante de cette collectivité d'une question relevant de sa compétence.

Dans les conditions prévues par la loi organique, les projets de délibération ou d'acte relevant de la compétence d'une collectivité territoriale peuvent, à son initiative, être soumis, par la voie du referendum, à la décision des électeurs de cette collectivité.

Lorsqu'il est envisagé de créer une collectivité territoriale dotée d'un statut particulier ou de modifier son organisation, il peut être décidé par la loi de consulter les électeurs inscrits dans les collectivités intéressées. La modification des limites des collectivités territoriales peut également donner lieu à la consultation des électeurs dans les conditions prévues par la loi.

Article 72-2. Les collectivités territoriales bénéficient de ressources dont elles peuvent disposer librement dans les conditions fixées par la loi.

Elles peuvent recevoir tout ou partie du produit des impositions de toutes natures. La loi peut les autoriser à en fixer l'assiette et le taux dans les limites qu'elle détermine.

Les recettes fiscales et les autres ressources propres des collectivités territoriales représentent, pour chaque catégorie de collectivités, une part déterminante de l'ensemble de leurs ressources. La loi organique fixe les conditions dans lesquelles cette règle est mise en œuvre.

Tout transfert de compétences entre l'Etat et les collectivités territoriales s'accompagne de l'attribution de ressources équivalentes à celles qui étaient consacrées à leur exercice. Toute création ou extension de compétences ayant pour conséquence d'augmenter les dépenses des collectivités territoriales est accompagnée de ressources déterminées par la loi.

La loi prévoit des dispositifs de péréquation destinés à favoriser l'égalité entre les collectivités territoriales.

Article 72-3. La République reconnaît, au sein du peuple français, les populations d'outre-mer, dans un idéal commun de liberté, d'égalité et de fraternité. 
La Guadeloupe, la Guyane, la Martinique, La Réunion, Mayotte, Saint-Pierre-etMiquelon, les îles Wallis et Futuna et la Polynésie française sont régis par l'article 73 pour les départements et les régions d'outre-mer et pour les collectivités territoriales créées en application du dernier alinéa de l'article 73, et par l'article 74 pour les autres collectivités.

Le statut de la Nouvelle-Calédonie est régi par le titre XIII.

La loi détermine le régime législatif et l'organisation particulière des Terres australes et antarctiques françaises.

Article 72-4. Aucun changement, pour tout ou partie de l'une des collectivités mentionnées au deuxième alinéa de l'article 72-3, de l'un vers l'autre des régimes prévus par les articles 73 et 74, ne peut intervenir sans que le consentement des électeurs de la collectivité ou de la partie de collectivité intéressée ait été préalablement recueilli dans les conditions prévues à l'alinéa suivant. Ce changement de régime est décidé par une loi organique.

Le Président de la République, sur proposition du Gouvernement pendant la durée des sessions ou sur proposition conjointe des deux assemblées, publiées au Journal officiel, peut décider de consulter les électeurs d'une collectivité territoriale située outre-mer sur une question relative à son organisation, à ses compétences ou à son régime législatif. Lorsque la consultation porte sur un changement prévu à l'alinéa précédent et est organisée sur proposition du Gouvernement, celui-ci fait, devant chaque assemblée, une déclaration qui est suivie d'un débat.

Article 73. Dans les départements et les régions d'outre-mer, les lois et règlements sont applicables de plein droit. Ils peuvent faire l'objet d'adaptations tenant aux caractéristiques et contraintes particulières de ces collectivités.

Ces adaptations peuvent être décidées par ces collectivités dans les matières où s'exercent leurs compétences et si elles y ont été habilitées par la loi.

Par dérogation au premier alinéa et pour tenir compte de leurs spécificités, les collectivités régies par le présent article peuvent être habilitées par la loi à fixer elles-mêmes les règles applicables sur leur territoire, dans un nombre limité de matières pouvant relever du domaine de la loi.

Ces règles ne peuvent porter sur la nationalité, les droits civiques, les garanties des libertés publiques, l'état et la capacité des personnes, l'organisation de la justice, le droit pénal, la procédure pénale, la politique étrangère, la défense, la sécurité et l'ordre publics, la monnaie, le crédit et les changes, ainsi que le droit électoral. Cette énumération pourra être précisée et complétée par une loi organique

La disposition prévue aux deux précédents alinéas n'est pas applicable au département et à la région de La Réunion. 
Les habilitations prévues aux deuxième et troisième alinéas sont décidées, à la demande de la collectivité concernée, dans les conditions et sous les réserves prévues par une loi organique. Elles ne peuvent intervenir lorsque sont en cause les conditions essentielles d'exercice d'une liberté publique ou d'un droit constitutionnellement garanti.

La création par la loi d'une collectivité se substituant à un département et une région d'outre-mer ou l'institution d'une assemblée délibérante unique pour ces deux collectivités ne peut intervenir sans qu'ait été recueilli, selon les formes prévues au second alinéa de l'article 72-4, le consentement des électeurs inscrits dans le ressort de ces collectivités.

Article 74. Les collectivités d'outre-mer régies par le présent article ont un statut qui tient compte des intérêts propres de chacune d'elles au sein de la République.

Ce statut est défini par une loi organique, adoptée après avis de l'assemblée délibérante, qui fixe :

les conditions dans lesquelles les lois et règlements y sont applicables ;

les compétences de cette collectivité ; sous réserve de celles déjà exercées par elle, le transfert de compétences de l'Etat ne peut porter sur les matières énumérées au quatrième alinéa de l'article 73 , précisées et complétées, le cas échéant, par la loi organique ;

les règles d'organisation et de fonctionnement des institutions de la collectivité et le régime électoral de son assemblée délibérante ;

les conditions dans lesquelles ses institutions sont consultées sur les projets et propositions de loi et les projets d'ordonnance ou de décret comportant des dispositions particulières à la collectivité, ainsi que sur la ratification ou l'approbation d'engagements internationaux conclus dans les matières relevant de sa compétence.

La loi organique peut également déterminer, pour celles de ces collectivités qui sont dotées de l'autonomie, les conditions dans lesquelles :

le Conseil d'Etat exerce un contrôle juridictionnel spécifique sur certaines catégories d'actes de l'assemblée délibérante intervenant au titre des compétences qu'elle exerce dans le domaine de la loi ;

l'assemblée délibérante peut modifier une loi promulguée postérieurement à l'entrée en vigueur du statut de la collectivité, lorsque le Conseil constitutionnel, saisi notamment par les autorités de la collectivité, a constaté que la loi était intervenue dans le domaine de compétence de cette collectivité ;

des mesures justifiées par les nécessités locales peuvent être prises par la collectivité en faveur de sa population, en matière d'accès à l'emploi, de droit d'établissement pour l'exercice d'une activité professionnelle ou de protection du patrimoine foncier ;

la collectivité peut participer, sous le contrôle de l'Etat, à l'exercice des compétences qu'il conserve, dans le respect des garanties accordées sur l'ensemble du territoire national pour l'exercice des libertés publiques. 
Les autres modalités de l'organisation particulière des collectivités relevant du présent article sont définies et modifiées par la loi après consultation de leur assemblée délibérante.

Article 74-1. Dans les collectivités d'outre-mer visées à l'article 74 et en NouvelleCalédonie, le Gouvernement peut, dans les matières qui demeurent de la compétence de l'Etat, étendre par ordonnances, avec les adaptations nécessaires, les dispositions de nature législative en vigueur en métropole, sous réserve que la loi n'ait pas expressément exclu, pour les dispositions en cause, le recours à cette procédure.

Les ordonnances sont prises en conseil des ministres après avis des assemblées délibérantes intéressées et du Conseil d'Etat. Elles entrent en vigueur dès leur publication. Elles deviennent caduques en l'absence de ratification par le Parlement dans le délai de dix-huit mois suivant cette publication.

\section{c) Bref commentaire des principaux articles révises}

\section{Articles concernant toutes les collectivités territoriales}

\section{Article 72-1 : Rôle accru des citoyens}

$>$ Les électeurs pourront demander par une pétition à leurs élus locaux de se saisir d'une question

$>$ Les électeurs pourront décider par referendum de la solution à apporter à une question qui leur sera posée par les élus locaux

$>$ Les électeurs pourront donner leur avis, à la demande du Parlement, lorsqu'il sera envisagé de créer pour eux une collectivité ayant un statut particulier ou bien lorsqu'il sera envisagé de modifier les limites de leur collectivité

Article 72-2 : Pouvoirs et moyens financiers des collectivités territoriales

$>$ Liberté de disposer de leurs ressources financières

$>$ Marge de manœuvre accrue en matière de fiscalité locale

$>$ Les ressources propres des collectivités territoriales doivent représenter une part "déterminante"

$>$ L'Etat doit transférer les ressources correspondant aux nouvelles compétences

$>$ Les inégalités entre les collectivités devront être compensées par la péréquation

\section{Articles concernant les collectivités territoriales situées outre-mer}

Article 72-3 : Enumération et classification des collectivités territoriales situées outre-mer 
$>$ Sont régis par l'article 73: les départements, les régions et les éventuelles collectivités uniques s'y substituant, situés en Guadeloupe, Guyane, Martinique et Réunion

$>$ Sont régies par l'article 74 : les collectivités d'outre-mer de Mayotte, Saint-Pierre-et-Miquelon, les îles Wallis et Futuna et la Polynésie française

$>$ Est régie par le Titre XIII de la Constitution : la Nouvelle-Calédonie

\section{Article 72-4 : Possibilités et modalités de changement}

$>$ Une collectivité située outre-mer peut passer du régime de l'article 73 à celui de l'article 74 et inversement

$>$ Le changement de régime est subordonné au consentement des électeurs concernés

$>$ Le Président de la République a l'initiative d'organiser la consultation des électeurs de la collectivité concernée sur les questions relatives à l'organisation, aux compétences ou au régime de leur collectivité 


\section{ANNEXE IV \\ LA DECLARATION DE BASSE-TERRE \\ Les régions d'outre-mer "Le courage politique au service du développement » \\ «DECLARATION FINALE »}

Les Présidents des régions de Guadeloupe, Guyane, Martinique, réunis à BasseTerre, le 01 décembre 1999, constatent :

- La dégradation continuelle depuis de nombreuses années de la situation de leurs Régions, illustrée notamment par :

- Une destruction systématique de l'initiative locale,

- Un affaiblissement des filières productives,

- La déstabilisation financière des Collectivités

- Un taux de chômage croissant (en moyenne 30\%).

- Le développement rapide des dérives sociales, destructrices pour leurs sociétés et en particulier pour les jeunes générations dont les perspectives d'emploi apparaissent de plus en plus aléatoires.

- L'impossibilité de mettre un terme à cette situation avec les moyens actuels qui leur sont dévolus dans le cadre de leurs compétences, malgré des efforts financiers importants des Collectivités Territoriales et les concours de l'Etat et de l'Union Européenne.

- L'inadéquation d'un dispositif fiscal et social conçu pour un pays développé et appliqué à des Régions en retard de développement.

Considérant les résultats obtenus par les autres Régions Ultrapériphériques (Canaries, Açores, Madère) en matière de développement et de progrès social.

\section{Décident :}

D'unir leurs efforts afin de bâtir un projet de développement économique social et culturel impliquant la prise en compte des identités propres à chaque Région et basé sur l'évidence que la dignité procède du travail et non de l'assistanat.

Ce projet doit encourager la créativité et l'émergence d'activités productives afin de mettre un terme au développement d'une «société de consommation ». 
Ce projet doit également rompre avec le cycle infernal de «l'emploi assisté » pour favoriser la création et le développement d'entreprises, orienter les jeunes vers les métiers à forte plus value, réaménager les formations vers les secteurs à forte potentialité.

Cela suppose aussi, un partenariat actif avec le secteur socioprofessionnel, qui doit participer à une nouvelle économie qui ne se réfère pas exclusivement à l'aide publique.

Les Présidents, conscients de la volonté de leur population de mieux maîtriser son devenir, affirment solennellement leur engagement à exposer et expliquer leur projet, afin de recueillir, après consultation, son adhésion.

En conséquence, les Présidents des trois Régions proposeront au Président de la République et au Gouvernement, une modification législative voire constitutionnelle, visant à créer un statut nouveau de Région d'Outre-Mer doté d'un régime fiscal et social spécial pour la Guadeloupe, la Guyane et la Martinique, dans le cadre de la République Française d'une part, et de l'Union Européenne d'autre part (article 299-2 du Traité d'Amsterdam).

Les Présidents des Régions de Guadeloupe, Guyane et Martinique réunis à BasseTerre le 1 décembre 1999, ont pour leur part ouvert le débat, défini les thèmes et proposé les orientations de ce chantier d'avenir, dans le respect des idéologies de chacun.

\section{Lucette MICHAUX-CHEVRY}

Le Président du Conseil Régional de la Guadeloupe Antoine KARAM

Le Président du Conseil Régional de la Guyane

Alfred MARIE-JEANNE

Le Président du Conseil Régional de la Martinique 